\title{
Direct WIMP searches with XENON100 and XENON1T
}

\author{
Ferella Alfredo Davide ${ }^{1, a}$, on behalf of the XENON collaboration \\ ${ }^{1}$ INFN - Laboratori Nazionali del Gran Sasso - Gran Sasso Science Institute, 67010 Assergi, Italy
}

\begin{abstract}
The XENON100 experiment is the second phase of the XENON direct Dark Matter search program. It consists of an ultra-low background double phase (liquid-gas) xenon filled time projection chamber with a total mass of $161 \mathrm{~kg}$ (62 in the target region and 99 in the active shield), installed at the Laboratori Nazionali del Gran Sasso (LNGS). Here the results from the 224.6 live days of data taken between March 2011 and April 2012 are reported. The experiment set one of the most stringent limits on the WIMPnucleon spin-independent cross section to date $\left(2 \times 10^{-45} \mathrm{~cm}^{2}\right.$ for a $55 \mathrm{Gev} / \mathrm{c}^{2}$ WIMP mass at $90 \%$ confidence level) and the most stringent on the spin-dependent WIMPneutron interaction $\left(3.5 \times 10^{-40}\right.$ for a $45 \mathrm{GeV} / \mathrm{c}^{2}$ WIMP mass $)$. With the same dataset, XENON100 excludes also solar axion coupling to electrons at $g_{A e}>7.7 \times 10^{-12}$ for a mass of $\mathrm{m}_{\text {Axion }}<1 \mathrm{keV} / \mathrm{c}^{2}$ and galactic axion couplings by $g_{A e}>1 \times 10^{-12}$ at a mass range of $\mathrm{m}_{\text {Axion }}=5-10 \mathrm{keV} / \mathrm{c}^{2}$ (both $90 \%$ C.L.). Moreover an absolute spectral comparison between simulated and measured nuclear recoil distributions of light and charge signals from a ${ }^{241} \mathrm{AmBe}$ source demonstrates a high level of detector and systematics understanding. Finally, the third generation of the XENON experiments, XENON1T, is the first tonne scale direct WIMP search experiment currently under construction. The commissioning phase of XENON1T is expected to start in early 2015 followed, a few months after, by the first science run. The experiment will reach sensitivities on the WIMP-nucleon spin-independent cross section down to $2 \times 10^{-47} \mathrm{~cm}^{2}$ after two years of data taking.
\end{abstract}

\section{Introduction}

An increasing number of astrophysical and astronomical observations points to the existence of a non luminous, non baryonic and cold (i.e. non relativistic) matter component of the Universe, therefore called Cold Dark Matter (CDM) [1-3]. The Dark Matter particles are still undetected, but they have to belong to a category beyond the Standard Model; the most appealing and interesting candidates are the so called Weakly Interactive Massive Particles (WIMPs) predicted by Supersymmetric theories (SUSY), models with extra dimensions and little Higgs models [4-6]. Different WIMP detection approaches are used in order to determine the characteristics of such particles: experiments at LHC will try to look for their appearance in the collisions; high energy astro-particle physics experiments are looking for WIMP annihilation signatures from the center of the Sun or from the center of the Galaxy and/or other places where the matter is quite dense whereas Dark Matter direct detection experiments are looking for the elastic scattering of WIMPs off the target nuclei.

\footnotetext{
ae-mail: ferella@lngs.infn.it
} 
Among the latter category, the XENON project is one of the most promising: after the results of a first $10 \mathrm{~kg}$ scale prototype, XENON10 [7], the second generation experiment (XENON100, the results of which are presented here) has been running since 2009 and successfully achieved its initial aim of improving the sensitivity by a factor $\sim 50$ with respect to XENON10. Similarly, the next phase of the program foresees the installation of a ton scale detector (XENON1T) aiming at a sensitivity 100 times better than that of XENON100. While XENON100 is still running, XENON1T is currently in advanced phase of construction at Laboratori Nazionali del Gran Sasso in Italy.

\section{The XENON100 experiment}

The use of liquid xenon (LXe) as target for a Dark Matter direct search experiment is motivated by several reasons: it has a high density $(\rho \sim 3 \mathrm{~g} / \mathrm{cm})$, easily allowing for scalability to ton scale detectors of relatively small sizes; has a high atomic number $(\mathrm{A} \sim 131)$ which enhances the probability of WIMP-nucleus spin-independent interactions $\left(\sigma \propto A^{2}\right)$; natural xenon has $\sim 50 \%$ abundance of odd isotopes, which allows to probe also the WIMP-nucleon spin dependent interactions; a low energy threshold is achievable thanks to the high ionization and scintillation yield in Liquid Xenon. Moreover the requirement of low background is fulfillable, given the absence of any long lived radioactive isotope in the noble liquid and considering its efficiency as self-shielding target, given the high Z (54). The latter feature, combined with the possibility of reconstructing the position of the interactions in $3 \mathrm{D}$ allows background reduction by volume fiducialization.

The XENON100 detector is a dual-phase (liquid-gas) Time Projection Chamber (TPC) filled with xenon: $\sim 62 \mathrm{~kg}$ of target volume surrounded by $\sim 99 \mathrm{~kg}$ of liquid xenon acting as a veto. The TPC is enclosed in a PTFE cylinder of $30 \mathrm{~cm}$ height and $\sim 30 \mathrm{~cm}$ diameter (PTFE is a suitable fluoropolymer with very good UV light reflective features [8]). The whole volume is viewed by a total of 242 squared $(1$ ” $\times 1$ ”) low radioactivity photo-multiplier tubes (PMTs) (Hamamatsu R8520-06-Al), especially developed to be operational at the Liquid Xenon temperature for the characteristic xenon scintillation wavelength $(\lambda=178 \mathrm{~nm}) .98$ of these PMTs are on the top of the TPC in the gas phase, arranged in concentric circles to improve position reconstruction, whereas 80 PMTs are on the bottom of the TPC placed very close to each other in order to optimize the light collection. The remaining 64 PMTs are placed in the veto, looking at the side, at the top and at bottom around the TPC.

The detector is located underground at the Laboratori Nazionali del Gran Sasso (LNGS) in Italy, under $1400 \mathrm{~m}$ (3600 meters water equivalent) of rock, where the muon flux is reduced by six orders of magnitude compared to the surface. All the detector construction materials were selected for low radioactivity [9] and cryogenic components and electrical feedthroughs were placed outside of a low radioactivity shield, made of $20 \mathrm{~cm}$ of lead, $20 \mathrm{~cm}$ of high density polyethylene and $5 \mathrm{~cm}$ of innermost copper.

The basic working principles of XENON100 are the following (see [10] for more details): a particle interacting in the Liquid Xenon target generates a prompt scintillation signal (S1) which is promptly detected by the two arrays of PMTs. The ionization electrons produced in the same interaction are drifted towards the anode, in an electric field across the TPC $(\sim 530 \mathrm{~V} / \mathrm{cm})$, and once they reach the liquid-gas interface, given the high $(\sim 12 \mathrm{kV} / \mathrm{cm})$ field in the $\sim 2.5 \mathrm{~mm}$ gas gap, such electrons are extracted from the liquid and accelerated in the gas, producing a secondary scintillation signal (S2), proportional to the ionization charge, which is also detected by the two arrays of PMTs. The time distance between S1 and S2 (drift time), since the electron drift velocity in liquid xenon is constant $(\sim 1.74 \mathrm{~mm} / \mu$ s at $0.53 \mathrm{kV} / \mathrm{cm})$, gives the information of the position of the interaction vertex in the vertical axis. Given that the diffusion of electrons in the liquid is very small and the S2 signal is very localized on the top, the hit pattern of the secondary scintillation signal in the top PMT array can 
be used in order to reconstruct the interaction vertex in the horizontal plane. Three different methods are used to reconstruct the $\mathrm{XY}$ position, based on $\chi^{2}$ minimization, neural network and support vector machine algorithms. In this way a 3-dimensional event position information is available. Moreover the nature of the impinging particles can be discriminated by studying the S1 and S2 signals; for electron recoils (usual background in the energy region of interest for Dark Matter search) the ratio of S2/S1 is much higher than for nuclear recoils (expected WIMP signal), because of the different ionization density of the two particles. This feature is used to discriminate between electronic recoil (ER) background events from nuclear recoil (NR) possibly signal events.

\section{The 2011/2012 science run}

The latest XENON100 dark matter data released, was taken in 13 months between March 2011 and April 2012[11]. Besides 3 major interruptions due to equipment maintenance, the detector ran continuously and the regular dark matter data taking was only interrupted by weekly PMT gain calibrations and electronic recoil (ER) calibrations, performed with ${ }^{60} \mathrm{Co}$ and ${ }^{232} \mathrm{Th}$ sources. The nuclear recoil (NR) calibration runs were taken at the beginning and at the end of the run, using a ${ }^{241} \mathrm{AmBe}$ neutron source. The exposure to WIMPs of this run is 224.6 live days. Dark matter data was blinded in order to avoid biases, in the $\mathrm{S} 1$ range $[2,100]$ photoelectrons $(\mathrm{PE})$.

With respect to the previous data set[12], this run has a longer exposure, a significantly lower intrinsic ${ }^{85} \mathrm{Kr}$ contamination ( $19 \pm 4$ ppt versus $450 \pm 30$ ppt of ${ }^{\text {nat }} \mathrm{Kr}$ ), an improved electronic noise condition and a lower trigger threshold ( $>99 \%$ for S2 signals bigger than $150 \mathrm{PE}$ ).

\subsection{The analysis}

The acceptance of all cuts has been calculated on NR calibration data, with the exception of the basic quality cuts, that might have a time dependence due to changing detector conditions. All the cuts were tested on both non-blind science data and ER calibration data. The cuts applied can be divided into two different categories: the basic quality cuts, devoted to eliminate non-physical and/or spurious events, due to noise or to interactions that happen in the gas phase etc... The second class of cuts identifies only those events compatible with the WIMP search analysis (i.e. single interactions in the detector), like single S1 and S2 signal selection and active veto coincidence events removal. Moreover an S2 threshold (> 150 PE) is applied along with the requirement that more than two PMTs in the TPC have to coincidently detect a physical signal, in order to ensure that only events well above the noise level are selected. Finally the analysis is applied only on $34 \mathrm{~kg}$ of active (fiducial) liquid xenon.

A profile likelihood statistical approach is used to test the background-only and signal hypotheses. As a cross-check a "benchmark" region between 6.6 and $30.5 \mathrm{keV}_{\mathrm{nr}}$ (corresponding to [3,20] PE) is defined on which a maximum gap counting method [13] is applied.

The expected background in the signal region is determined by ER events (majority of the events originating from detector construction materials and LXe intrinsic radioactivity) leaking into the NR benchmark region $(0.79 \pm 0.16)$ and by NR from neutrons $(0.17+0.12-0.07)$. The former class of background events is estimated from ${ }^{60} \mathrm{Co}$ and ${ }^{232} \mathrm{Th}$ source data ( $>35$ times more statistics than in the science data), while the latter from Monte Carlo simulation, with inputs from both cosmic muon spectrum and momentum distribution in the underground location of the experiment and the radioactivity of the detector construction and surrounding materials[14].

\subsection{The results}

WIMPs in the halo of our Galaxy are non-relativistic and their interactions with nuclei can be characterized in terms of scalar (or spin-independent, SI) and axial-vector (or spin-dependent, SD) couplings 
$[15,16]$. In the case of SI interactions, the leading contribution of the scattering is coherent across the nucleus, and roughly scales with $A^{2}$, where $A$ is the number of nucleons. On the other hand, if the WIMP is a spin- $1 / 2$ or a spin- 1 field, the contributions to the WIMP-nucleus scattering cross section arise from couplings of the WIMP field to the quark axial current, i.e. it couples only to nuclei with odd number of protons and/or neutrons.

After unblinding, two events were found in the benchmark region. This led to the $90 \%$ confidence level (CL) exclusion on spin-independent WIMP-nucleon cross section reported in Fig. 1. Standard assumptions on the astrophysical parameters were made: isothermal WIMP halo with a local density of $\rho_{\chi}=0.3 \mathrm{GeV} / \mathrm{cm}^{3}$ [17], a local circular velocity of $v_{0}=220 \mathrm{~km} / \mathrm{s}$, and a Galactic escape velocity of $v_{\text {esc }}=544 \mathrm{~km} / \mathrm{s}$ [18]. Systematic uncertainties in the energy scale as described by the $\mathcal{L}_{\text {eff }}$ parametrization of [12] and in the background expectation are "profiled out" and represented in the limit. Poisson fluctuations in the number of PEs dominate the S1 energy resolution and are also taken into account along with the single PE resolution. The new limit is represented by the thick blue line in Fig. 1 and is the best limit to date, and starts scanning regions preferred by the constrained supersymmetric parameter space [19].

In XENON100, the isotopic abundances of odd isotope nuclei, ${ }^{129} \mathrm{Xe}(\operatorname{spin} 1 / 2)$ and ${ }^{131} \mathrm{Xe}$ (spin $3 / 2$ ), are $26.2 \%$ and $21.8 \%$, respectively. Using the profile likelihood approach mentioned above, constraints on the spin-dependent WIMP-nucleon cross section were derived [20], as shown in Figure 2 for neutron couplings along with results from other experiments. The $1 \sigma(2 \sigma)$ uncertainty on the sensitivity of this run is shown as a green (yellow) band.

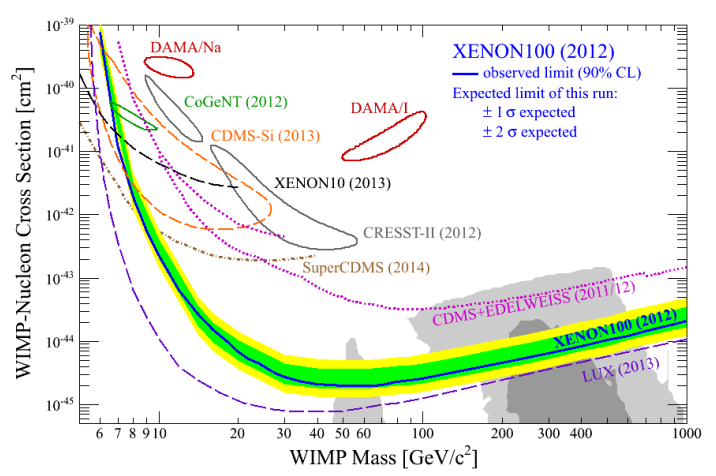

Figure 1. Result on the spin-independent WIMPnucleon cross section from XENON100 [11]. The expected sensitivity of this run is shown by the green/yellow band $(1 \sigma / 2 \sigma)$ and the resulting exclusion limit (90\% CL) in blue. For comparison, other experimental limits (90\% CL) and detection claims $(2 \sigma)$ are also shown [21-25], together with the regions $(1 \sigma / 2 \sigma)$ preferred by supersymmetric (CMSSM) models [19].

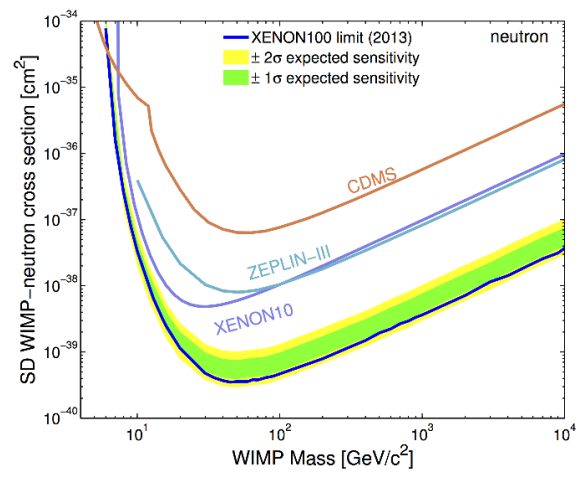

Figure 2. 90\% CL upper limit on the spin-dependent WIMP-neutron cross section from XENON100 [20]. The $1 \sigma(2 \sigma)$ uncertainty on the expected sensitivity of this run is show as a green (yellow) band. Also shown are results from XENON10 [26], CDMS [27, 28], ZEPLIN-III [29], PICASSO [30] , COUPP [31], SIMPLE [32], KIMS [33], IceCube [34] in the hard $\left(W^{+} W^{-}, \tau^{+} \tau^{-}\right.$for WIMP masses $\left.<80.4 \mathrm{GeV} / \mathrm{c}^{2}\right)$ and soft $(b \bar{b})$ annihilation channels. 


\subsection{Solar Axions and ALPs}

Axions were introduced by Peccei and Quinn to solve the strong CP problem as pseudo-NambuGoldstone bosons emerging from the breaking of a global U(1) symmetry [35-37]. Although this original model has been ruled out, "invisible" axions arising from a higher symmetry-breaking energy scale are still allowed, as described, for example, in the DFSZ and KSVZ models [38-41]. In addition to QCD axions, axion-like particles (ALPs) are pseudoscalars that do not necessarily solve the strong CP problem, but that were introduced by many extensions of the Standard Model of particle physics. Axions as well as ALPs are another class of well motivated cold dark matter candidates [42].

Astrophysical observations are thought to be the most sensitive technique for detecting axions and ALPs [43]: the Sun would constitute an intense source of this particles (referred to as solar axions), where they can be produced via Bremsstrahlung, Compton scattering, axio-recombination and axiodeexcitation [44]. Additionally, searches can be conducted for ALPs that may have been generated via a non-thermal production mechanism in the early Universe and which now constitute the dark matter in our galaxy (referred to as galactic ALPs).

Axions and ALPs may give rise to observable signatures in detectors through their coupling to photons $\left(g_{A \gamma}\right)$, electrons $\left(g_{A e}\right)$ and nuclei $\left(g_{A N}\right)$. The coupling $g_{A e}$ may be tested via scattering off the electrons of a target, such as liquid xenon (LXe), through the axio-electric effect [45-49]. This process is the analogue of the photo-electric effect with the absorption of an axion instead of a photon.

The first axion searches performed with the XENON100 experiment are reported in [50]. The expected interaction rate is obtained by the convolution of the flux and the axio-electric cross section. The latter is given, both for QCD axions and ALPs, by [46-49, 51]:

$$
\sigma_{A e}=\sigma_{p e}\left(E_{A}\right) \frac{g_{A e}{ }^{2}}{\beta_{A}} \frac{3 E_{A}^{2}}{16 \pi \alpha_{e m} m_{e}^{2}}\left(1-\frac{\beta_{A}^{2 / 3}}{3}\right),
$$

where $\sigma_{p e}$ is the photoelectric cross section for LXe [52], $E_{A}$ is the axion energy, $\alpha_{e m}$ is the fine structure constant, $m_{e}$ is the electron mass, and $\beta_{A}$ is the axion velocity over the speed of light, $c$.

For solar axions, both flux (taken from [44]) and cross-section depend upon $g_{A e}^{2}$, thus the interaction rate scales with the fourth power of the coupling. For the ALPs, assuming that they constitute the whole dark matter halo density $\left(\rho_{D M} \sim 0.3 \mathrm{GeV} / \mathrm{cm}^{3}[17]\right)$, the total flux is given by $\phi_{\mathrm{ALP}}=c \beta_{A} \times \rho_{D M} / m_{A}$, where $m_{A}$ is the ALP mass. The interaction rate for these ALPs depends on $g_{A e}^{2}$, as the flux is independent from the axion coupling. As $\beta_{A} \approx 10^{-3}$ in the non-relativistic regime, the velocities cancel out in the convolution between $\sigma_{A e}$ and the flux. Thus the expected electron recoil spectrum is independent from the particle speed and a monoenergetic peak at the axion mass is expected in the spectrum.

Fig. 3 shows the new XENON100 exclusion limit on $g_{A e}$ at 90\% CL. Within the DFSZ and KSVZ models [38-41] XENON100 excludes axion masses above $0.3 \mathrm{eV} / \mathrm{c}^{2}$ and $80 \mathrm{eV} / \mathrm{c}^{2}$, respectively. For comparison, the CAST experiment, testing the coupling to photons, $g_{A \gamma}$, has excluded axions within the KSVZ model in the mass range between $0.64-1.17 \mathrm{eV} / \mathrm{c}^{2}[59,60]$.

The XENON100 90\% CL exclusion limit for galactic ALPs is shown in Fig. 4, together with other experimental constraints $[53,61,62]$. In the $5-10 \mathrm{keV} / \mathrm{c}^{2}$ mass range, XENON100 sets the best upper limit, excluding an axion-electron coupling $g_{A e}>1 \times 10^{-12}$ at the $90 \% \mathrm{CL}$, assuming that ALPs constitute all of the galactic dark matter.

\section{Detector response to nuclear recoils}

An absolute spectral comparison between Monte Carlo (MC) simulation of the neutron exposure and the equivalent calibration data from a ${ }^{241} \mathrm{AmBe}(\alpha, n)$ neutron source has been carried out [63]. Scin- 


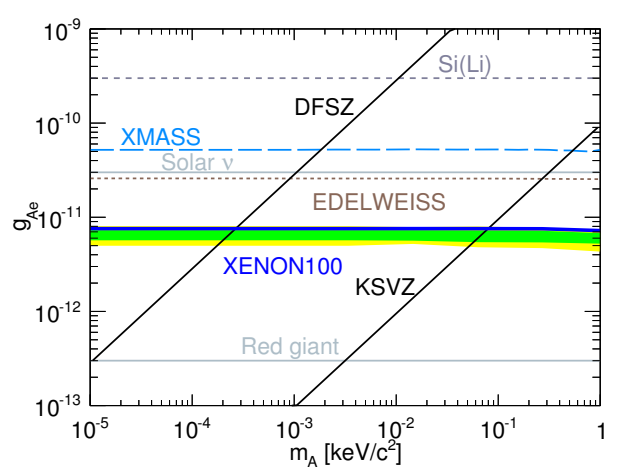

Figure 3. The XENON100 limits ( $90 \%$ CL) on solar axions(blue line) [50]. The expected sensitivity is shown by the green/yellow bands $(1 \sigma / 2 \sigma)$. Limits by EDELWEISS-II [53], and XMASS [54] are shown, together with the limits from a $\mathrm{Si}(\mathrm{Li})$ detector from Derbin et al. [55]. Indirect astrophysical bounds from solar neutrinos [56] and red giants [57] are represented by dashed lines. The benchmark DFSZ and KSVZ models are represented by grey dashed lines [38-41].

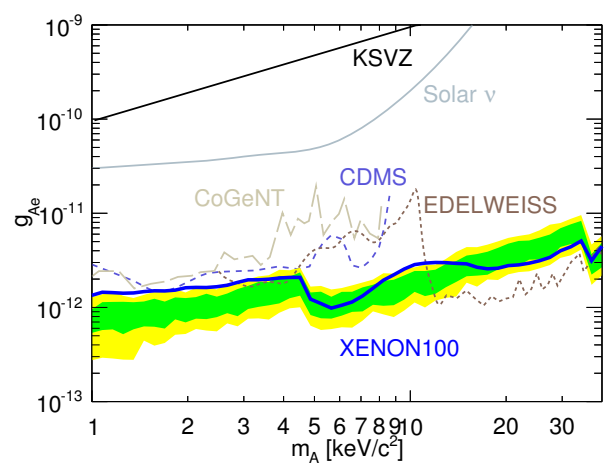

Figure 4. The XENON100 limit (90\% CL) on ALP coupling to electrons as a function of the mass (blue line) [50]. The expected sensitivity is shown by the green/yellow bands $(1 \sigma / 2 \sigma)$. The other curves are constraints set by CoGeNT [61] (brown dashed line), CDMS [62] (grey continuous line), and EDELWEISSII [53] (red line, extending up to $40 \mathrm{keV} / \mathrm{c}^{2}$ ). Indirect astrophysical bound from solar neutrinos [56] is represented as a dashed line. The benchmark KSVZ model is represented by a dashed grey line $[40,41]$.

tillation and ionization channels have been defined independently to compare $\mathrm{S} 1, \mathrm{~S} 2$ and $\log _{10}(\mathrm{~S} 2 / \mathrm{S} 1)$ (i.e. the discrimination parameter). This allows the parameterization of both the charge yield $Q_{\mathrm{y}}$ (i.e. the number of ionization electrons produced by a neutron recoil of a given energy) and the relative scintillation efficiency $\mathcal{L}_{\text {eff }}$ (the energy dependent yield for scintillation photons emitted following a nuclear recoil). The two quantities are needed to convert from observed S1 and S2 signals to true recoil energy deposition, respectively. In Fig. 5 and 6 the absolute comparison between MC and data $\mathrm{S} 1$ and S2 spectra is shown, respectively. A good agreement is achieved for both channels throughout the entire energy ranges.

\section{XENON1T}

The XENON1T detector is a scaled up design of the successful XENON100 one: it is a dual-phase liquid xenon time projection chamber (TPC), containing 3.3 tons ( 2.2 tons active volume) of highpurity liquid xenon, instrumented from both above and below with low-radioactivity photomultiplier tubes and kept under a uniform electric drift field of about $1.0 \mathrm{kV} / \mathrm{cm}$.

As in the previous iteration of the XENON project, XENON1T has been designed to provide an improvement in sensitivity with respect to the previous experiment by almost two orders of magnitude. In order to fulfill such a challenging requirement a detector with a larger target mass and lower background levels have been designed, based on the expertise developed within the collaboration through the years and on specific and dedicated R\&Ds. 


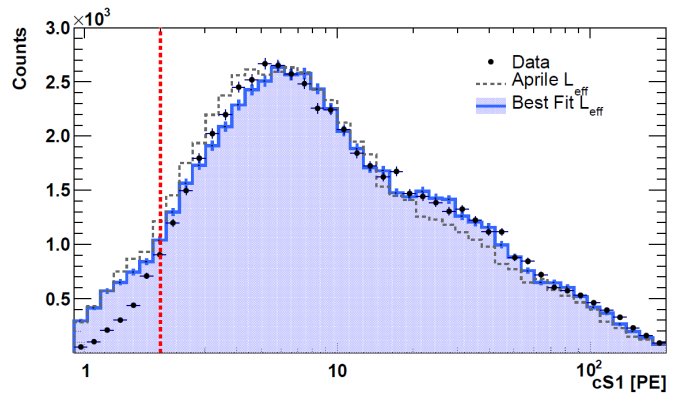

Figure 5. Fit of the simulated $\mathrm{S} 1$ spectrum to data (black points) [63]. The MC spectrum (blue) is obtained using the $\mathcal{L}_{\text {eff }}$ after the optimization process. Reasonable agreement between data and MC above $2 \mathrm{PE}$ is achieved.

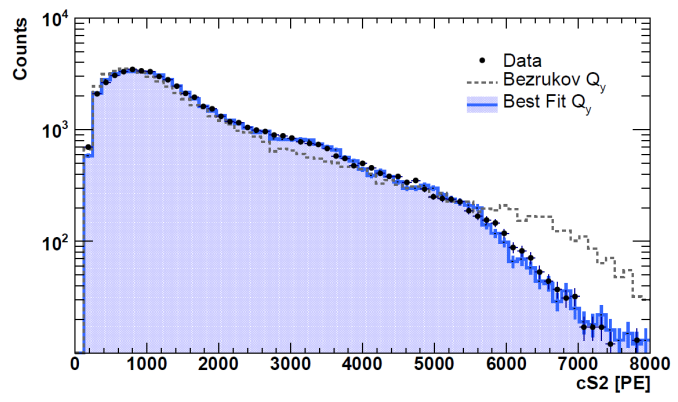

Figure 6. Comparison between the MC and data S2 spectra [63]. The black data-points indicate the data and the blue spectrum is obtained as the result of the optimization of $Q_{\mathrm{y}}$. Good agreement across the whole signal range is achieved.

With a larger target mass there are more benefits both in terms of sensitivity and in background reduction due to self-shielding. However there exist technical challenges that put a limit on this parameter; in particular the main one is the fact that a stable $1 \mathrm{kV} / \mathrm{cm}$ electric field has to be achieved; therefore the detector was designed with a maximum drift distance of 1 meter. In order to test the feasibility of such a relatively long maximum drift length, a XENON1T "demonstrator" has been operated at Columbia University in order to test the cryogenic and circulation systems at large drift lengths.

The need for an ultra-low background in the central detector region requires the installation of the detector underground, inside a shield that attenuates the environmental radiation; moreover an extensive screening and selection of all detector construction materials is being carried out. Another important source of nuclear recoil backgrounds are the cosmic muons which produce neutrons as secondary products when passing near the detector. Therefore the detector will be installed in the center of a large cylindrical tank filled up with ultra pure water (acting as a shield against environmental radioactivity) and instrumented with 84 PMTs that turn it into a veto that detects cosmic muons and cosmogenic hadronic particles [64]. A non-negligible contribution to the overall XENON1T background comes from intrinsic contamination of the xenon: commercial xenon contains krypton on the ppb level. The unstable isotope ${ }^{85} \mathrm{Kr}$ comprises only a factor of $\sim 2 \times 10^{-11}$ of natural krypton but its $\beta$-decays cause a significant background for a dark matter detector. Such impurities within the xenon volume itself are not rejected by fiducialization or analysis and must be removed physically. For XENON1T a krypton distillation column is used to remove krypton impurities from the xenon. This reduces levels of krypton to below the ppt level (target $0.5 \times 10^{-12} \mathrm{Kr} / \mathrm{Xe}$ ). The other intrinsic contamination to the xenon is the ${ }^{222} \mathrm{Rn}$ emanating from ${ }^{226} \mathrm{Ra}$ implanted in the surfaces of the materials in direct contact with the xenon. In order to reduce also this kind of radioactivity methods are being employed for the reduction of the surface ${ }^{226} \mathrm{Ra}$ contamination and A predicted background of 0.15 events/year in the WIMP search region originates from impurities in the xenon. Finally the irreducible background constituted by the solar neutrinos and the double beta decays of ${ }^{136} \mathrm{Xe}$ (about $10 \%$ of natural xenon) contribute an estimated 0.09 events/year. 
The full background prediction from all sources is about 0.5 events/year.

\section{Conclusion}

While XENON100 is still running, the XENON1T detector construction in the Hall B of the Gran Sasso Underground Laboratory (LNGS), started in the fall of 2013, will continue until mid of 2015. Detector commissioning and a first science run are expected by 2015, while the full dark matter sensitivity will be reached by 2017 , after two years of operation. With realistic assumptions on the detector performance and analysis efficiency, the maximal sensitivity to spin-independent WIMPnucleon cross sections is expected to be $2 \times 10^{-47} \mathrm{~cm}^{2}$ at a WIMP mass around $40 \mathrm{GeV} / \mathrm{c}^{2}$.

\section{References}

[1] W. Freedman and M. Turner, Rev. Mod. Phys., 75, 1433 (2003).

[2] D. Clowe et al., ApJ, 648, L109 (2006).

[3] M.J. Jee et al., http://xxx.lanl.gov/abs/0705.2171

[4] A. Bottino et al., Phys. Rev.D69 037302 (2004);

[5] J. Ellis et al., Phys. Rev.D71 095007 (2005);

[6] H. C. Cheng et al., Phys. Rev. Lett. 89211301 (2002);

[7] J. Angle et al. (XENON10 Collaboration), Phys. Rev. Lett. 100, 021303 (2008).

[8] M. Yamashita et al., NIM A 535, 692 (2004).

[9] E. Aprile et al. [XENON100 Collaboration], Astropart. Phys. 35 (2011) 43

[10] E. Aprile et al. [XENON100 Collaboration], Astropart. Phys. 35 (2012) 573

[11] E. Aprile et al. [XENON100 Collaboration], Phys. Rev. Lett. 109 (2012) 181301 [arXiv:1207.5988 [astro-ph.CO]].

[12] E. Aprile et al. [XENON100 Collaboration], Phys. Rev. Lett. 107 (2011) 131302

[13] S. Yellin, Phys. Rev. D 66 (2002) 032005

[14] E. Aprile et al. [XENON100 Collaboration], J. Phys. G: Nucl. Part. Phys. 40, 115201 (2013), arXiv: 1306.2303.

[15] M. W. Goodman and E. Witten, Phys. Rev. D 31 (1985) 3059.

[16] G. Jungman, M. Kamionkowski and K. Griest, Phys. Rept. 267 (1996) 195 [hep-ph/9506380].

[17] A. M. Green, Mod. Phys. Lett. A27, 1230004 (2012).

[18] M. C. Smith et al., Mon. Not. R. Astron. Soc. 379, 755 (2007).

[19] Combined region using C. Strege et al., JCAP 1203, 030 (2012); A. Fowlie et al. (2012), arXiv: 1206.0264; O. Buchmueller et al. (2011), arXiv: 1112.3564.

[20] E. Aprile et al. [XENON100 Collaboration], Phys. Rev. Lett. 111 (2013) 021301 arXiv: 1301.6620

[21] C. Savage et al., JCAP 0904, 010 (2009).

[22] C. E. Aalseth et al. (CoGeNT), Phys. Rev. Lett. 106, 131301 (2011).

[23] G. Angloher et al. (CRESST-II), Eur. Phys. J. C72, 1971 (2012).

[24] Z. Ahmed et al. (CDMS), Science 327, 1619 (2010); Z. Ahmed et al. (CDMS), Phys. Rev. Lett. 106, 131302 (2011); E. Armengaud et al. (EDELWEISS), Phys. Lett. B 702, 329 (2011); J. Angle et al. (XENON10), Phys. Rev. Lett. 107, 051301 (2011); M. Felizardo et al. (SIMPLE), Phys. Rev. Lett. 108, 201302 (2012); E. Behnke et al. (COUPP) (2012), arXiv: 1204.3094; D. Y. Akimov et al. (ZEPLIN-III), Phys. Lett. B 709, 14 (2012); E. Armengaud et al. (EDELWEISS) (2012), arXiv: 1207 . 1815. 
[25] D. S. Akerib et al. [LUX Collaboration], Phys. Rev. Lett. 112 (2014) 9, 091303 [arXiv:1310.8214 [astro-ph.CO]].

[26] J. Angle et al. (XENON10), Phys. Rev. Lett. 101, 091301 (2008), arXiv:0805.2939.

[27] Z. Ahmed et al. (CDMS), Phys. Rev. Lett. 102, 011301 (2009).

[28] Z. Ahmed et al. (CDMS-II), Phys. Rev. Lett. 106, 131302 (2011), arXiv:1011.2482.

[29] D. Akimov et al. (ZEPLIN-III), Phys.Lett. B709, 14 (2012), arXiv:1110.4769.

[30] S. Archambault et al. (PICASSO) (2012), arXiv:1202.1240.

[31] E. Behnke et al. (COUPP) (2012), arXiv:1204.3094.

[32] M. Felizardo et al. (SIMPLE) (2011), arXiv:1106.3014.

[33] S. Kim, H. Bhang, J. Choi, W. Kang, B. Kim, et al., Phys.Rev.Lett. 108, 181301 (2012), arXiv: 1204.2646.

[34] M. G. Aartsen et al. (IceCube collaboration) (2012), arXiv:1212.4097.

[35] R. D. Peccei and H. R. Quinn, Phys. Rev. Lett. 1440, 38 (1977).

[36] S. Weinberg, Phys. Rev. Lett. 40, 223 (1978).

[37] F. Wilczeck, Phys. Rev. Lett. 40, 279 (1978).

[38] M. Dine, W. Fischler, and M. Srednicki, Phys. Lett B 104, 199 (1981).

[39] A. R. Zhitnitsky, Sov. J. Nucl. Phys. 31, 260 (1980).

[40] J. E. Kim, Phys. Rev. Lett. 43, 103 (1979).

[41] M. A. Shifman, A. I. Vainshtein, and V. I. Zakharov, Nucl. Phys. B 166, 493 (1980).

[42] L. Abbott and P. Sikivie, Phys. Lett. B120, 133 (1983).

[43] P. Sikivie, Phys. Rev. Lett. 51, 1415 (1983).

[44] J. Redondo (2013), arXiv:1310.0823.

[45] S. Dimopoulos, G. D. Starkman, and B. W. Lynn, Phys. Rev. B 168, 145 (1986).

[46] F. T. Avignone et al., Phys. Rev. D 35, 2752 (1987).

[47] M. Pospelov, A. Ritz, and M. Voloshin, Phys. Rev. D 78, 115012 (2008).

[48] A. Derevianko et al., Phys. Rev. D 82, 065006 (2010).

[49] K. Arisaka et al., Astropart. Phys. 44, 59 (2013).

[50] E. Aprile et al. (XENON100 Coll.), Phys. Rev. D 90 (2014) 062009, arXiv:1404.1455.

[51] F. Alessandria et al. (CUORE Coll.), JCAP 1305, 007 (2013).

[52] NIST, URL http://physics.nist.gov/PhysRefData/Xcom/html/xcom1.html.

[53] E. Armengaud et al. (EDELWEISS-II Coll.), JCAP 1311, 067 (2013).

[54] K. Abe et al. (XMASS Coll.), Phys. Lett. B 724, 46 (2013).

[55] A. Derbin et al., JETP Lett. 95, 339 (2012).

[56] P. Gondolo and G. G. Raffelt, Phys. Rev. D 79, 107301 (2009).

[57] N. Viaux, M. Catelan, P. B. Stetson, G. G. Raffelt, J. Redondo, A. A. R. Valcarce, and A. Weiss, Phys. Rev. Lett. 111, 231301 (2013).

[58] G. G. Raffelt, Lect. Notes Phys. 741, 51 (2008).

[59] M. Arik et al. (CAST Coll.), Phys. Rev. Lett. 112, 091302 (2014).

[60] K. Barth et al. (CAST Coll.), JCAP 1305, 010 (2013), arXiv:1302.6283.

[61] C. E. Aalseth et al. (CoGeNT Coll.), Phys. Rev. Lett. 101, 251301 (2008).

[62] Z. Ahmed et al. (CDMS Coll.), Phys. Rev. Lett. 103, 141802 (2009).

[63] E. Aprile et al. [XENON100 Collaboration], Phys. Rev. D 88 (2013) 012006 arXiv: 1304.1427.

[64] E. Aprile et al. [XENON100 Collaboration], 2014 JINST 9 P11006 arXiv : 1406.2374 